\title{
Time domain equalization method for TS-OFDM signal under higher mobile environments
}

\author{
Pongsathorn Reangsuntea ${ }^{1 *}{ }^{*}$, Pisit Boonsrimuang ${ }^{2}$, Kazuo Mori $^{1}$ and Hideo Kobayashi ${ }^{1 \dagger}$
}

\begin{abstract}
In higher time-varying fading channels, the signal quality of orthogonal frequency division multiplexing (OFDM) technique would be degraded relatively due to the occurrence of inter-carrier interference (ICI). To solve this problem, this paper firstly proposes a new design of time domain training sequence (TS) in the estimation of channel impulse response (CIR) for the TS-OFDM signal which can reduce the leakage of power spectrum density (PSD) at the outside of OFDM allocated frequency bandwidth with keeping higher CIR estimation accuracy. Secondly, this paper proposes a time domain equalization (TDE) method which can achieve better bit error rate (BER) performance with keeping lower computation complexity even in higher time-varying fading channels. The salient feature of the proposed TDE method is to employ a partial differentiation for the time domain CIR matrix for solving the maximum likelihood (ML) equation in which the time domain CIR matrix becomes a symmetric banded matrix. From this feature, a low-complexity parallel block inverse matrix algorithm can be employed in the calculation of inverse matrix in keeping the same accuracy as that of the direct inverse matrix calculation. This paper presents various computer simulation results to demonstrate the effectiveness of the proposed TDE method for the TS-OFDM signal as compared with conventional frequency domain equalization (FDE) methods under higher mobile environments.
\end{abstract}

Keywords: TS-OFDM, Time domain equalization (TDE), Inter-carrier interference (ICI), Time-varying fading channels

\section{Introduction}

Orthogonal frequency division multiplexing (OFDM) has been employed as an efficient transmission technique in various wireless communications systems which require the reliable high data rate communications in the frequency-selective multipath fading channels [1]. Up to today, OFDM has been adopted as the standard transmission technique in the digital audio broadcasting (DAB), digital video broadcasting (DVB), wireless local area network (WLAN), and the fourth-generation (4G) cellular systems [2]. The salient feature of OFDM technique is to employ the guard interval (GI) for avoiding the intersymbol interference (ISI) with keeping the orthogonality among OFDM subcarriers even in multipath fading channels. To realize the property of this function, the

\footnotetext{
${ }^{*}$ Correspondence: pongsathorn@com.elec.mie-u.ac.jp

${ }^{\dagger}$ Equal contributor

'Department of Systems Engineering, Graduate School of Engineering, Mie University, 514-8507 Mie, Japan

Full list of author information is available at the end of the article
}

cyclic prefix OFDM (CP-OFDM), zero padding OFDM (ZP-OFDM), and time domain training sequence inserted OFDM (TS-OFDM) techniques were proposed in the transmission of OFDM signal [3]. The CP-OFDM employs the cyclic prefix $(\mathrm{CP})$ as the role of $\mathrm{GI}$ to avoid the ISI. The ZP-OFDM employs the zero padding between OFDM data symbols as the GI which enables the reduction of transmission power. The TS-OFDM employs the pre-designed time domain training sequence such as a pseudo-noise (PN) sequence [4] or Chu sequence [5] as the GI besides being used in the channel estimation.

In the OFDM signal under the static or lower mobile environments, the channel impulse response (CIR) can be assumed to be the time-invariant within one OFDM symbol period. From this fact, the simple frequency domain equalization (FDE) method with one-tap filter can be used to compensate the distortion of multipath fading. However, the CIR is no more constant even within one OFDM symbol period in higher time-varying fading channels which are experienced by the users on the high speed
Springer Open

(C) The Author(s). 2017 Open Access This article is distributed under the terms of the Creative Commons Attribution 4.0 International License (http://creativecommons.org/licenses/by/4.0/), which permits unrestricted use, distribution, and reproduction in any medium, provided you give appropriate credit to the original author(s) and the source, provide a link to the Creative Commons license, and indicate if changes were made. 
trains or vehicles. In higher time-varying fading channels, the orthogonality among OFDM subcarriers is no more satisfied which leads to the fatal degradation of bit error rate (BER) performance due to the occurrence of inevitable inter-carrier interference (ICI) caused by the higher Doppler frequency spread [6].

To solve the above problem, many frequency domain equalization methods were proposed to mitigate the ICI in higher time-varying channels [7-14]. These methods are designated as the frequency domain equalization (FDE) in which the equalization processing for the mitigation of ICI is conducted in the frequency domain. In $[7,8]$, the ICI self-cancellation methods were proposed to mitigate ICI at the cost of degradation in the transmission data rate. In [9], the FDE method with the minimum mean square error (MMSE-FDE) and successive interference cancellation (SIC) equalization methods were proposed for the CP-OFDM signal. These methods are required to employ the direct inverse matrix calculation for the channel frequency response (CFR) matrix in solving the simultaneous equations. However, the computation complexity for the inverse matrix calculation required at every symbol is relatively higher which leads to a serious problem in the implementation of practical receiver. To solve this problem, [10] proposed a low-complexity MMSE-FDE method for CP-OFDM signal with the parallel block inverse matrix algorithm in the calculation of inverse matrix [15]. In this method, the full elements of CFR matrix is approximated by a banded matrix so as to enable the employment of parallel block inverse matrix algorithm. However, the BER performance would be degraded in higher time-varying fading channels due to the approximation for the full elements of CFR matrix by the banded matrix. In [11], the fast sub-optimal FAST-MMSE-FDE method was proposed for the ZP-OFDM signal under the assumption of perfect channel knowledge. Although this method can achieve lower complexity by assuming the linear changing of CIR within one OFDM symbol period, it has a difficulty to estimate the CFR matrix precisely by using the pilot subcarriers in the frequency domain because the received pilot subcarriers are also affected by the ICI in higher time-varying fading channels [12].

To solve the above problem on the CFR estimation by using the pilot subcarriers for the CP-OFDM and ZP-OFDM signals in higher time-varying fading channels, a PN time domain sequence is employed as the TS in the estimation of CFR as well as the role of GI for the TS-OFDM signal [13]. In [13], since an overlap and add frequency domain equalization (OLA-FDE) method is employed, the property of circular convolution is no more satisfied in higher time-varying fading channels which leads to the fatal degradation of BER performance. In [14], the low-complexity OLA-MMSE-FDE and the FAST-MMSE-FDE methods were proposed for the TS-OFDM signal. These methods enable the considerable reduction of computation complexity by using the Maclaurin's expansion approximation technique in the calculation of inverse matrix under the assumption that the CIR is changing linearly within one symbol period. However, the approximation of linear changing of CIR is no more effective in higher time-varying fading channels which leads to the degradation of BER performance.

The employment of TS-OFDM signal with the time domain PN or Chu sequence can achieve higher CFR estimation accuracy than the CP-OFDM and ZP-OFDM signals of using the pilot subcarriers in higher time-varying fading channels. However, the employment of PN or Chu sequence as the TS would cause the undesirable leakage of power spectrum density (PSD) at the outside of the allocated OFDM frequency bandwidth. Because the occupied frequency bandwidth of TS signal would be much wider than the allocated OFDM frequency bandwidth when the TS signal is employed so as to keep the good property of autocorrelation for the $\mathrm{PN}$ or Chu sequences which leads to the serious adjacent channel interference problem to other systems [16]. Furthermore, when the same data pattern of $\mathrm{PN}$ or Chu sequence is added at every data symbol over one frame, higher power spurious components would occur at the outside of the allocated OFDM frequency bandwidth due to the repetition of same data pattern of TS signal in the time domain. The leakage of PSD at the outside of OFDM allocated bandwidth would cause the difficulty in the design of transmitter so as to meet the spectral mask requirement for the adjacent channel interference. From this fact, the leakage of PSD must be suppressed when using the TS-OFDM signal.

From the above backgrounds, this paper firstly proposes the channel estimation method by using a new design of TS signal. The feature of proposed channel estimation method are to employ a different data pattern of TS signal added to each data symbol over one frame and to employ the triangular window function for the TS signal as the waveform shaping both for reducing the leakage of PSD with keeping higher CIR estimation accuracy. Secondly, this paper proposes an equalization method of using the CIR matrix in the time domain instead of using the CFR matrix for the conventional FDE methods. Hereafter, the proposed method is designated as the time domain equalization (TDE) in which the equalization processing for the mitigation of ICI is conducted in the time domain. The feature of the proposed TDE method is to employ the partial differentiation for the time domain CIR matrix for solving the maximum likelihood (ML) equation in which the time domain CIR matrix becomes a symmetric banded matrix. This feature enables to employ the parallel block inverse matrix algorithm which can achieve the same BER performance with much lower computation complexity as compared with that of the direct inverse matrix 
calculation [15]. These are completely different features from the conventional FDE methods of which CFR matrix is the full elements of matrix. The proposed TDE method can also obtain the frequency diversity gain at the fading deep dips [17] which enables to achieve better BER performance than the conventional FDE methods of using the CFR matrix as referred above [7-14].

The remainder of this paper is organized as follows. Section 2 firstly proposes a new design of TS signal for the CIR estimation method and secondly proposes the TDE method with the parallel block inverse matrix algorithm for the TS-OFDM signal. Section 3 evaluates the overall computation complexity for the proposed TDE method as compared with the various conventional FDE methods. Section 4 presents various computer simulation results to verify the effectiveness of the proposed TDE method for the TS-OFDM signal as compared with various conventional FDE methods for both the CP-OFDM and TS-OFDM signals. Finally, Section 5 draws some conclusions.

\section{Proposal of CIR estimation and time domain equalization methods}

This section presents the system model for the proposed TDE method and proposes a new design of TS signal by using a time domain triangular window function for the TS-OFDM signal so as to reduce the leakage of PSD. This section also proposes the TDE method of using a low-complexity parallel block inverse matrix algorithm which can achieve better BER performance than the conventional FDE methods with keeping lower complexity even in higher time-varying fading channels.

\subsection{Proposed TS-OFDM system model}

Figure 1 shows the structure of transceiver for the proposed TDE method for the TS-OFDM signal. At the transmitter, the input data information is modulated by the quadrature-phase shift keying (QPSK) or quadratureamplitude modulation (QAM) method in the frequency domain. The modulated signal $X(m, k)$ at the $k$ th subcarrier of the $m$ th symbol is converted to the time domain signal by $N$-point inverse fast Fourier transform (IFFT) which can be given by

$$
\begin{gathered}
x(m, n)=\frac{1}{\sqrt{N}} \sum_{k=0}^{N-1} X(m, k) e^{j \frac{2 \pi n k}{N}}, \\
0 \leq n \leq N-1
\end{gathered}
$$

where $x(m, n)$ is the transmitted time domain OFDM signal at the $n$th time sample of $m$ th symbol.

Figure 2a shows the frame format for the proposed TSOFDM signal with the time domain triangular window function. In the proposed frame format, the time domains TS1 and TS2 with the length of $S$ samples are inserted at the start of every data symbol in which the length of $S$ should be taken longer than the number of delay paths $(L)$ occurring in the multipath fading channels. The time domain TS would be used in the estimation of CIR at every sampling time and also used as the role of GI to avoid the inter-symbol interference (ISI).

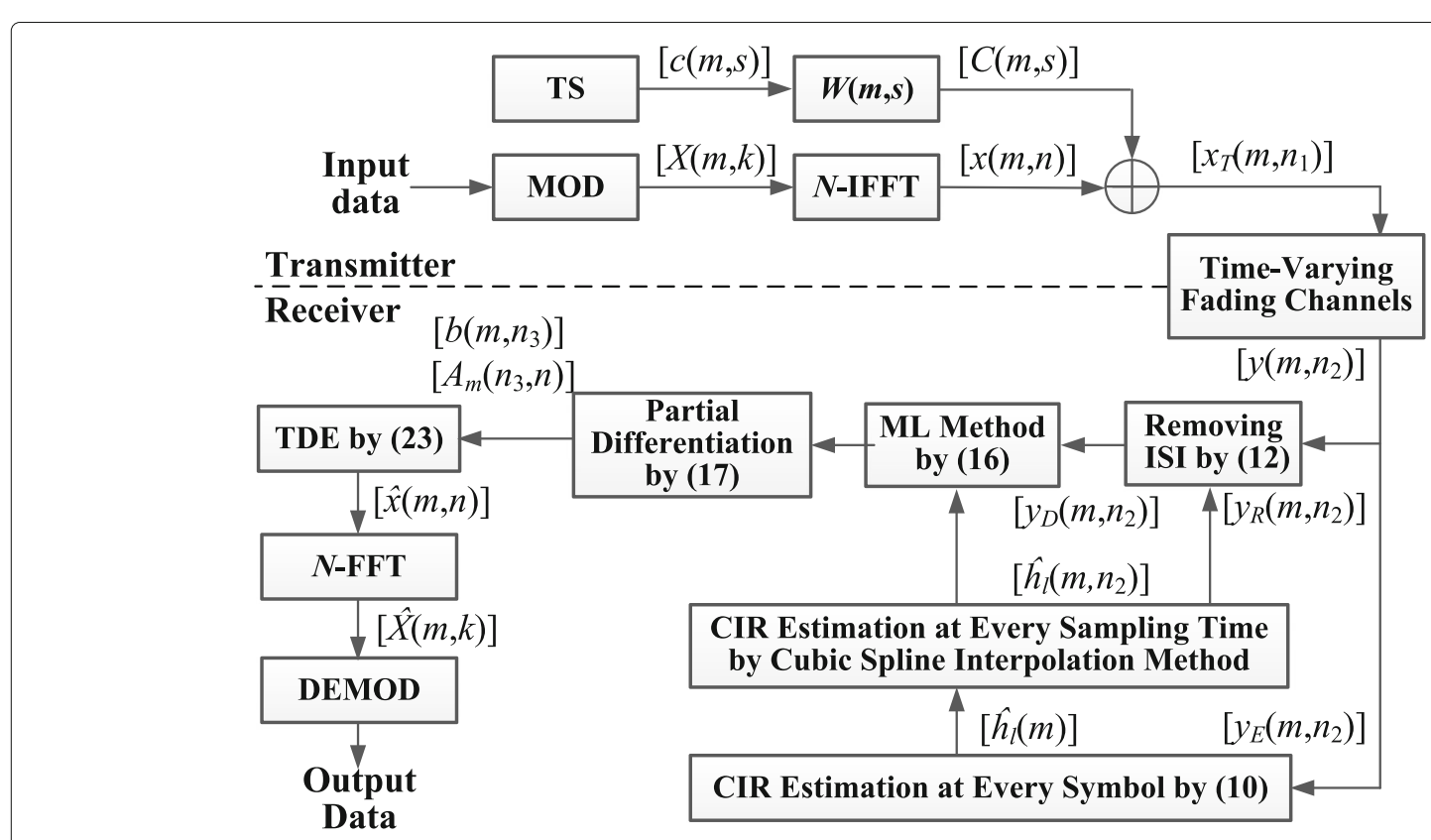

Fig. 1 Structure of transceiver for proposed TDE method 


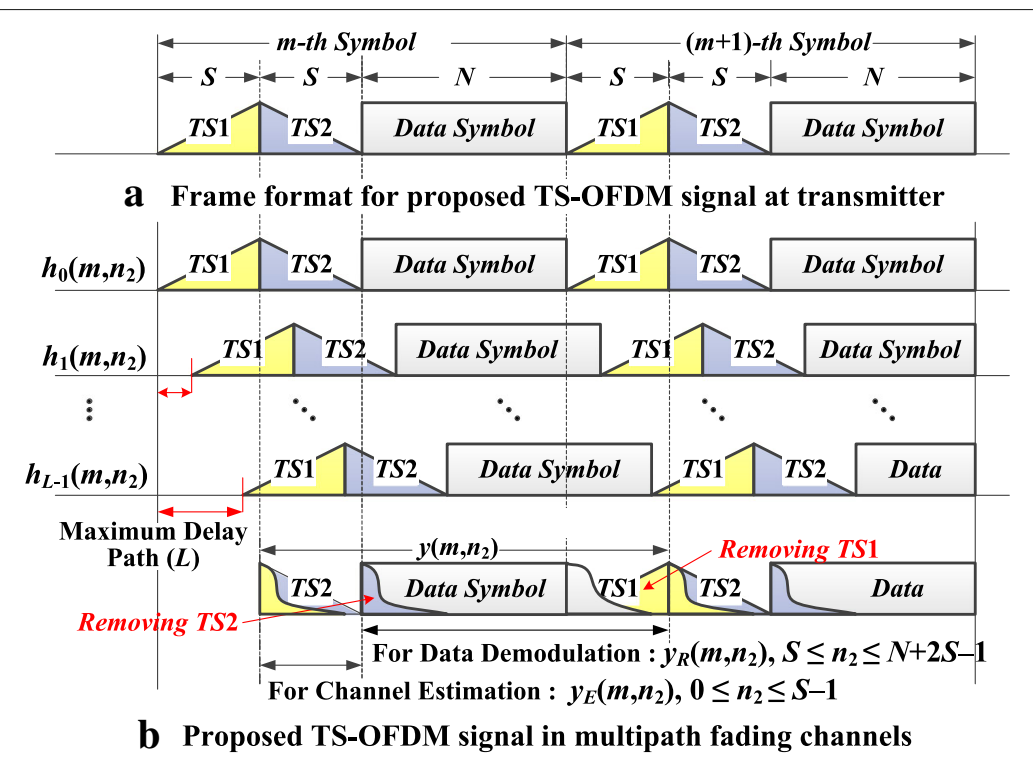

Fig. 2 Structure of frame format for TS-OFDM signal at transmitter and receiver. a Frame format for proposed TS-OFDM signal at transmitter. b Proposed TS-OFDM signal in multipath fading channels

The leakage of power spectrum density (PSD) for the TS-OFDM signal would occur due to the repetition of the same data pattern of TS signal over one frame and also due to the discontinuities at both ends of TS signal in the time domain. To reduce the leakage of PSD due to the repetition of the same data pattern of TS signal, this paper employs a different data pattern of TS signal for each data symbol over one frame as shown in Fig. 2a. The different data patterns of TS signals over one frame are generated by using some part of time domain OFDM signal of which occupied bandwidth even when employing some part of OFDM signal is basically the same as that using the time domain OFDM signal. The different data patterns of TS signals which can achieve higher CIR estimation accuracy in the multipath fading channels are selected by using the computer simulation results. In the computer simulations, the TS data patterns having higher CIR estimation accuracy are selected by changing the randomly generated data patterns of OFDM symbols which are independent from the data pattern of transmission information data symbols. It should be noted that the selected data patterns of TS signals can achieve higher CIR estimation accuracy even when these TS signals are added at the start of random information data symbols. In the implementation of practical systems, all system parameters including the number of data symbols in one frame are known in advance. From this fact, it is possible to prepare the required number of TS data patterns in advance which can achieve higher CIR estimation accuracy by using the computer simulation results.
As for the discontinuities at the both ends of TS signal in the time domain, this paper employs the time domain triangular window function for the TS signal as the waveform shaping. The window function is commonly used in the digital processing to reduce the leakage of PSD. The triangular window function is one of window functions such as the Hamming, Hann (raised cosine) and Blackman windows. It is well known that these window functions can improve the leakage of PSD by shaping the time domain signal by taking zero at both ends of signal of which various window functions are discussed theoretically in [18]. In this paper, we employ the triangular window function for the TS signal because of its simpler waveform. By using the proposed TS signals having the different data patterns over one frame in conjunction with the time domain triangular window function, the leakage of PSD could be suppressed relatively.

In this paper, the data pattern for the proposed TS1 and TS2 signals at the $m$ th symbol employs the same pattern which is represented by $c(m, s)$. Furthermore, the TS1 and TS2 signals at the $m$ th data symbol are multiplied by the time domain triangular window function of $W(s)$ to suppress the leakage of PSD as shown in Fig. 1. The time domain triangular window function $W(s)$ with the length of $2 S$ samples is given by

$$
W(s)= \begin{cases}(s+1) / S, & 0 \leq s \leq S-1 \\ 2-(s / S), & S \leq s \leq 2 S-1\end{cases}
$$


The proposed TS1 and TS2 signals at the $m$ th data symbol after multiplying $W(s)$ can be expressed by

$$
C(m, s)=\left\{\begin{array}{c}
d_{1}(m, s)=c(m, s) W(s) \\
0 \leq s \leq S-1 \\
d_{2}(m, s)=c(m, s-S) W(s) \\
S \leq s \leq 2 S-1,
\end{array}\right.
$$

where $C(m, s)$ with the length of $2 S$ samples is the sequential data pattern of TS1 and TS2 from 0 to $2 S-1$ in the time domain. Then, $C(m, s)$ is inserted at the start of every OFDM data symbol which is represented by

$$
x_{T}\left(m, n_{1}\right)=\left\{\begin{array}{l}
C\left(m, n_{1}\right), \\
0 \leq n_{1} \leq 2 S-1 \\
x\left(m, n_{1}-2 S\right), \\
2 S \leq n_{1} \leq N+2 S-1,
\end{array}\right.
$$

where $x_{T}\left(m, n_{1}\right)$ is the transmission data signal including the TS signal at the $n_{1}$ th sample of $m$ th symbol with the length of $N+2 S$ samples. Assuming that the synchronization of symbol timing at the receiver is perfect, the received time domain OFDM signal $y\left(m, n_{2}\right)$ at the $n_{2}$ th sampling time of $m$ th symbol after passing through the multipath fading channels as shown in Fig. 2b can be expressed by

$$
\begin{aligned}
y\left(m, n_{2}\right)= & \sum_{l=0}^{L-1} h_{l}\left(m, n_{2}\right) x_{T}\left(m, n_{2}-l\right) \\
& +z\left(m, n_{2}\right), 0 \leq n_{2} \leq N+2 S-1,
\end{aligned}
$$

where $h_{l}\left(m, n_{2}\right)$ is the CIR at the $n_{2}$ th time sample of $m$ th data symbol for the $l$ th delay path occurred in the actual time-varying fading channels, $z\left(m, n_{2}\right)$ is the additive white Gaussian noise (AWGN) with the variance of $\sigma^{2}$, and $L$ is the number of delay paths. As shown in Figs. 1 and $2 \mathrm{~b}$, the received signal $y\left(m, n_{2}\right)$ can be divided into two parts which consist of the observation period for the CIR estimation $y_{E}\left(m, n_{2}\right)$ with the length of $S$ samples and for the data demodulation period $y_{R}\left(m, n_{2}\right)$ with the length of $P=N+S-1$ samples, respectively.

\subsection{Proposed CIR estimation method at every sampling time}

The received time domain TS2 signal $y_{E}\left(m, n_{2}\right)$ which is used in the estimation of CIR at every symbol can be expressed by

$$
\begin{aligned}
y_{E}\left(m, n_{2}\right)= & \sum_{l=0}^{L-1} h_{l}\left(m, n_{2}\right) d_{2}\left(m, n_{2}-l\right) \\
& +z\left(m, n_{2}\right), 0 \leq n_{2} \leq S-1,
\end{aligned}
$$

where $d_{2}\left(m, n_{2}\right)$ is the TS2 signal with the triangular window function given in (3). Here, it should be noted that the CIR is assumed to be constant during the short time period of TS1 and TS2 even in higher time-varying fading channels. Assuming $\hat{h}_{l}(m)$ is the unknown parameter for the CIR at the $m$ th symbol, the expected received time domain TS2 signal $\hat{y}_{E}\left(m, n_{2}\right)$ can be given by

$$
\begin{aligned}
\hat{y}_{E}\left(m, n_{2}\right)= & \sum_{l=0}^{S-1} h_{l}(m) d_{2}\left(m, n_{2}-l\right), \\
& 0 \leq n_{2} \leq S-1 .
\end{aligned}
$$

Here it should be noted that $d_{2}\left(m, n_{2}\right)$ given in (3) is known at the receiver and the number of delay paths to be estimated is assumed by $S$ of which length is taken by longer than the number of delay paths $L$ because the actual number of delay paths $L$ is unknown at the receiver. The unknown parameter of CIR $\hat{h}_{l}(m)$ at the $m$ th symbol can be estimated by solving the following maximum likelihood (ML) equation under the constraint for minimizing the difference between the actual received TS signal $y_{E}\left(m, n_{2}\right)$ in (6) and the expected received TS signal $\hat{y}_{E}\left(m, n_{2}\right)$ in (7) [19].

$$
\Gamma=\underset{\hat{h}_{l}(m)}{\arg \min }\left[\sum_{n_{2}=0}^{S-1}\left|y_{E}\left(m, n_{2}\right)-\hat{y}_{E}\left(m, n_{2}\right)\right|^{2}\right]
$$

By using a simple solution of (8) when assuming $y_{E}\left(m, n_{2}\right)=\hat{y}_{E}\left(m, n_{2}\right)$, the ML equation in (8) can be represented by the following simultaneous equations.

$$
\left[y_{E}\left(m, n_{2}\right)\right]_{S \times 1}=\left[d_{2}\left(m, n_{2}-l\right)\right]_{S \times S}\left[\hat{h}_{l}(m)\right]_{S \times 1}
$$

The unknown parameter of CIR $\hat{h}_{l}(m)$ at the $m$ th symbol can be solved by the following matrix calculation of using the inverse matrix of $\left[d_{2}\left(m, n_{2}-l\right)\right]_{S \times S}$.

$$
\left[\hat{h}_{l}(m)\right]_{S \times 1}=\left[d_{2}\left(m, n_{2}-l\right)\right]_{S \times S}^{-1}\left[y_{E}\left(m, n_{2}\right)\right]_{S \times 1},
$$

where $[\cdot]^{-1}$ represents the matrix inversion. Since all elements of $\left[d_{2}\left(m, n_{2}-l\right)\right]$ given in (3) are known at the receiver, the inverse matrix in (10) can be calculated in advance for all different data patterns of TS signals inserted over one frame which enables the considerable reduction of computation complexity in the estimation of CIR at every symbol.

By using the estimated $\hat{h}_{l}(m)$ at every symbol, the CIR at every sampling time $\hat{h}_{l}\left(m, n_{2}\right)$ can be estimated by using the cubic spline interpolation method over one frame. The PSD at the output of transmitter and CIR estimation accuracy at every sampling time $\hat{h}_{l}\left(m, n_{2}\right)$ for the proposed TS-OFDM signal is evaluated in Section 4. 


\subsection{Proposed time domain equalization method}

Considering the observation period for the data demodulation $y_{R}\left(m, n_{2}\right)$ as shown in Fig. $2 \mathrm{~b}$, the received time domain data signal can be given by

$$
\begin{aligned}
y_{R}\left(m, n_{2}\right)= & \sum_{l=0}^{L-1} h_{l}\left(m, n_{2}\right) x_{T}\left(m, n_{2}-l\right) \\
& +z\left(m, n_{2}\right), S \leq n_{2} \leq N+2 S-1,
\end{aligned}
$$

where $x_{T}\left(m, n_{2}\right)$ is the transmitted time domain signal given in (4) and $h_{l}\left(m, n_{2}\right)$ is the time domain CIR at every sampling time in the actual time-varying fading channels. From Fig. 2b, it can be observed that the received signal in (11) includes the ISI from $d_{2}(m, s)$ (TS2 of current symbol) and $d_{1}(m+1, s)$ (TS1 of next symbol). By using the estimated $\operatorname{CIR} \hat{h}_{l}\left(m, n_{2}\right)$ at every sampling time and the known data pattern of TS1 and TS2 with the time domain triangular window function given in (3), the ISI from the time domain TS at the both ends of data symbol can be removed by using the following equation.

$$
y_{D}\left(m, n_{2}\right)=\left\{\begin{array}{c}
y_{R}\left(m, n_{2}\right)-\sum_{l=n_{2}-S+1}^{S-1} \hat{h}_{l}\left(m, n_{2}\right) . \\
d_{2}\left(m, n_{2}-l\right) \\
S \leq n_{2} \leq 2 S-2 \\
y_{R}\left(m, n_{2}\right), \\
2 S-1 \leq n_{2} \leq N+S-1 \\
y_{R}\left(m, n_{2}\right)-\sum_{l=0}^{n_{2}-N-S} \hat{h}_{l}\left(m, n_{2}\right) . \\
d_{1}\left(m+1, n_{2}-N-S-l\right), \\
N+S \leq n_{2} \leq N+2 S-2
\end{array}\right.
$$

In the derivation of (12), the following relationships are used.

$$
\begin{aligned}
& n_{2}-l \leq S-1, \\
& \quad x_{T}\left(m, n_{2}-l\right)=d_{2}\left(m, n_{2}-l\right) \\
& n_{2}-l \geq N+S, \\
& \quad x_{T}\left(m, n_{2}-l\right)=d_{1}\left(m+1, n_{2}-N-S-l\right),
\end{aligned}
$$

where $y_{D}\left(m, n_{2}\right)$ in (12) is the received time domain signal after removing the ISI from the TS. The received time domain signal $y_{D}\left(m, n_{2}\right)$ over the observation period with $P(=N+S-1)$ samples which includes the time domain data symbol and TS1 after removing the ISI can be used in the proposed TDE. By using the observation period of $P$ samples including the TS signal, the proposed TDE method can achieve the frequency diversity gain at the fading deep dips [13] which could achieve better BER performance than the conventional FDE methods with the observation period of $N$ samples both under the quasi-static and higher mobile environments [17].
When the transmitted time domain data signal $\hat{x}(m, n)$ is assumed as the unknown parameters, the expected received signal $\hat{y}_{D}\left(m, n_{2}\right)$ without the ISI which corresponds to the actual received signal in (12) can be expressed by

$$
\hat{y}_{D}\left(m, n_{2}\right)=\left\{\begin{array}{c}
\sum_{l=0}^{n_{2}-S} \hat{h}_{l}\left(m, n_{2}\right) \hat{x}\left(m, n_{2}-S-l\right), \\
S \leq n_{2} \leq 2 S-2 \\
\sum_{l=0}^{S-1} \hat{h}_{l}\left(m, n_{2}\right) \hat{x}\left(m, n_{2}-S-l\right), \\
2 S-1 \leq n_{2} \leq N+S-1 \\
S-1 \quad \hat{h}_{l}\left(m, n_{2}\right) . \\
\sum_{l=n_{2}-N-S+1} \\
\hat{x}\left(m, n_{2}-S-l\right), \\
N+S \leq n_{2} \leq N+2 S-2 .
\end{array}\right.
$$

The unknown parameter of time domain data signal $\hat{x}(m, n)$ which corresponds to the transmitted time domain data given in (1) can be estimated by solving the following ML equation under the constraint for minimizing the difference between the actual received signal $y_{D}\left(m, n_{2}\right)$ in (12) and the expected received signal $\hat{y}_{D}\left(m, n_{2}\right)$ in (15).

$$
\Gamma=\underset{\hat{x}(m, n)}{\arg \min }\left[\sum_{n_{2}=S}^{N+2 S-2}\left|y_{D}\left(m, n_{2}\right)-\hat{y}_{D}\left(m, n_{2}\right)\right|^{2}\right]
$$

The ML equation in (16) can be transformed to the simultaneous equations by taking the partial differentiation for the unknown parameters $\hat{x}^{*}\left(m, n_{3}\right),\left(0 \leq n_{3} \leq\right.$ $N-1$ ) which can be expressed by

$$
\begin{aligned}
& \frac{\partial \Gamma}{\partial \hat{x}^{*}\left(m, n_{3}\right)}= \\
& \quad \frac{\partial\left[\sum_{n_{2}=S}^{N+2 S-2}\left|y_{D}\left(m, n_{2}\right)-\hat{y}_{D}\left(m, n_{2}\right)\right|^{2}\right]}{\partial \hat{x}^{*}\left(m, n_{3}\right)}=0,
\end{aligned}
$$

where $(\cdot)^{*}$ represents the conjugate complex. By using (17), the ML equation in (16) can be expressed by the following simultaneous equations with $N$ unknown time domain data information of $[\hat{x}(m, n)]$.

$$
\left[b\left(m, n_{3}\right)\right]_{N \times 1}=\left[A_{m}\left(n_{3}, n\right)\right]_{N \times N}[\hat{x}(m, n)]_{N \times 1},
$$


where $\left[b\left(m, n_{3}\right)\right]$ and $\left[A_{m}\left(n_{3}, n\right)\right]$ are the received time domain signal and the CIR matrix both after the partial differentiation, respectively, which are given by

$$
\begin{aligned}
{\left[b\left(m, n_{3}\right)\right]_{N \times 1}=} & \sum_{n_{2}=S}^{N+2 S-2} y_{D}\left(m, n_{2}\right) \frac{\partial \hat{y}_{D}^{*}\left(m, n_{2}\right)}{\partial \hat{x}^{*}\left(m, n_{3}\right)} \\
= & {\left[\hat{h}_{l}^{H}\left(m, n_{3}\right)\right]_{N \times P}\left[y_{D}\left(m, n_{2}\right)\right]_{P \times 1}, } \\
& 0 \leq n_{3} \leq N-1 \\
{\left[A_{m}\left(n_{3}, n\right)\right]_{N \times N}=} & \sum_{n_{2}=S}^{N+2 S-2} \hat{y}_{D}\left(m, n_{2}\right) \frac{\partial \hat{y}_{D}^{*}\left(m, n_{2}\right)}{\partial \hat{x}^{*}\left(m, n_{3}\right)} \\
= & {\left[\hat{h}_{l}^{H}\left(m, n_{3}\right)\right]_{N \times P}\left[\hat{h}_{l}\left(m, n_{2}\right)\right]_{P \times N}, } \\
& 0 \leq n_{3} \leq N-1,
\end{aligned}
$$

where $(\cdot)^{H}$ is the Hermitian transposition. In the deviation of (19) and (20), the following relationships are used.

$$
\begin{aligned}
& n_{3}=n_{2}-S-l \text { in } \frac{\partial \hat{y}_{D}^{*}\left(m, n_{2}\right)}{\partial \hat{x}^{*}\left(m, n_{3}\right)} \\
& n=n_{2}-S-l \text { in } \hat{y}_{D}\left(m, n_{2}\right)
\end{aligned}
$$

From (18), the unknown parameters $[\hat{x}(m, n)]$ can be solved by using the inverse matrix of $\left[A_{m}\left(n_{3}, n\right)\right]$ which can be given by

$$
[\hat{x}(m, n)]_{N \times 1}=\left[A_{m}\left(n_{3}, n\right)\right]_{N \times N}^{-1}\left[b\left(m, n_{3}\right)\right]_{N \times 1} .
$$

From (23), the proposed TDE method is required for the inverse matrix calculation which leads relatively higher computation complexity with the order of $O\left[N^{3}\right]$. To solve this problem, this paper proposes a low-complexity TDE method by using the good property of time domain CIR matrix $\left[A_{m}\left(n_{3}, n\right)\right]$ given after the partial differentiation. From (20), the CIR matrix $\left[A_{m}\left(n_{3}, n\right)\right]$ can be represented by

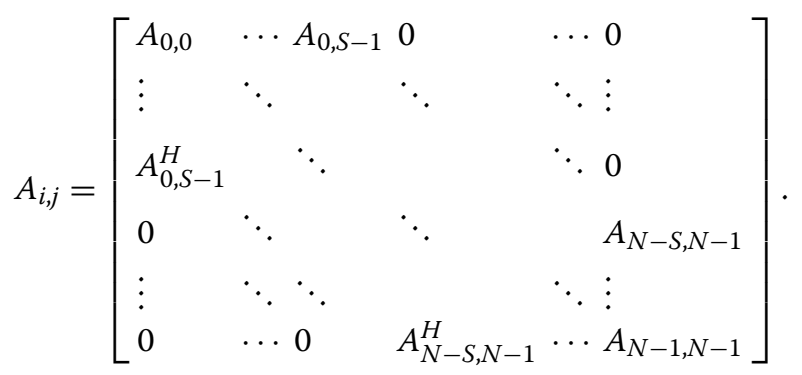

In (24), $A_{m}(i, j)$ in (20) is represented by $A_{i, j}$ and the index of the $m$ th symbol is omitted for brevity. From (24), it can be seen that the matrix is the banded matrix with the upper and lower bandwidth $\left(Q_{1}\right)$ of length $(S-1)$ whose non-zero entries are confined to a diagonal bands. Also the lower band matrix with the index of $(j, i)$ below the diagonal terms is the Hermitian transpose of upper band matrix with index of $(i, j)$. From these features, the CIR matrix $\left[A_{m}\left(n_{3}, n\right)\right]$ after the partial differentiation becomes the symmetric banded matrix with the block size of $(S \times S)$. From this fact, it is possible to employ the lowcomplexity parallel block inverse matrix algorithm [15] for solving (23). This feature of time domain CIR matrix for the proposed TDE method is completely different from the frequency domain CFR matrix for the conventional FDE methods of which CFR matrix is the full elements of matrix $[3,7-12,14]$. Here it should be noted that although the CFR matrix of the conventional MMSE-FDE method for the CP-OFDM signal is not the banded matrix, it is possible to employ the parallel block inverse matrix algorithm by assuming that the elements in the CFR matrix both at the lower and upper sides of diagonal bands are small enough [10]. However, this assumption would be no more effective in higher time-varying fading channels because the elements both at the lower and upper sides of diagonal bands in the CFR matrix are unable to be approximated as the negligible quantity. This means that the conventional MMSE-FDE method with the parallel block inverse matrix algorithm in [10] can achieve the reduction of computation complexity at the cost of considerable BER degradation in higher time-varying fading channels.

In [15], the parallel block inverse matrix algorithm is proposed for the symmetric banded matrix which can calculate the inverse matrix as the same accuracy as that for the direct inverse matrix calculation with much lower computation complexity. The key idea of this algorithm is to construct the small hierarchy of Schur complement blocks and then extracting the inverse matrix of diagonal hierarchy of Schur complement blocks. In (24), the size of small hierarchy of Schur complement blocks is equal to the length of TS $(=S)$. By using this algorithm, the inverse matrix of (24) can be calculated perfectly with the same accuracy as that for the direct inverse matrix calculation. The order of computation complexity required in the calculation of inverse matrix for the banded matrix $\left[A_{m}\left(n_{3}, n\right)\right]$ with the size of $(N \times N)$ can be reduced to $O\left[3 N S^{2}\right]$ by employing the parallel block inverse matrix algorithm. This complexity is much smaller than that for the direct inverse matrix calculation which requires the complexity $O\left[N^{3}\right]$. The overall computation complexities for the proposed TDE and conventional FDE methods are evaluated in Section 3.

By converting the estimated time domain data information $[\hat{x}(m, n)]$ in $(23)$ to the frequency domain by using $N$-point FFT, the frequency domain data information $[\hat{X}(m, k)]$ can be obtained and demodulated as shown in Fig. 1. 


\section{Evaluation of computation complexity}

This section evaluates the computation complexity for the proposed TDE method. Table 1 shows the order of complexities for the proposed TDE method when using the direct inverse matrix calculation and the low-complexity parallel block inverse matrix algorithm. In the table, the conventional MMSE-FDE method for the CP-OFDM $[9,10]$, the OLA-MMSE-FDE method for the TS-OFDM, and the FAST-MMSE-FDE method for the TS-OFDM [14] are also shown for the purpose of comparisons with the proposed TDE method.

In the proposed TDE method for the TS-OFDM with the parallel block inverse matrix algorithm, the removal of ISI incurred both ends of data symbol from the TS given in (12) requires the complexity $O\left[S^{2}-S\right]$. The construction of the time domain received signal $\left[b\left(m, n_{3}\right)\right]$ and the CIR matrix $\left[A_{m}\left(n_{3}, n\right)\right]$ with the partial differentiation given in (19) and (20) require the complexity $O[N S]$ and $O\left[N S^{2}\right]$, respectively. The parallel block inverse matrix algorithm for the symmetric banded matrix $\left[A_{m}\left(n_{3}, n\right)\right]$ given in (24) requires the complexity $O\left[3 N S^{2}\right]$ [15]. The multiplication of $\left[A_{m}\left(n_{3}, n\right)\right]^{-1}$ and $\left[b\left(m, n_{3}\right)\right]$ matrixes which corresponds to the time domain equalization to obtain the time domain signal $[\hat{x}(m, n)]$ in (23) requires the complexity $O\left[N^{2}\right]$. Finally, $N$-point FFT to $[\hat{x}(m, n)]$ for obtaining the frequency domain data information $[\hat{X}(m, k)]$ requires the complexity $\mathrm{O}\left[\mathrm{Nlog}_{2} \mathrm{~N}\right]$.

In the conventional MMSE-FDE method for the CPOFDM [10], the CFR matrix is approximated by the banded matrix with the block size $\left(Q_{1}+1\right) \times\left(Q_{1}+\right.$ 1 ), where $Q_{1}$ is the lower and upper bandwidth of approximated CFR banded matrix. The construction of the approximated banded CFR matrix with the upper and lower bandwidth of $Q_{1}$ and the received frequency domain signal matrix including one $N$-point FFT require the complexity $O\left[\left(2 Q_{1}+2\right) N \log _{2} N+N\left(Q_{1}+1\right)^{2}+N\left(Q_{1}+\right.\right.$ 1) $\left.+\mathrm{Nlog}_{2} N\right]$. The parallel block inverse matrix algorithm for the approximated CFR banded matrix with the bandwidth of $Q_{1}$ requires the complexity $O\left[3 N\left(Q_{1}+1\right)^{2}\right]$. Finally, the frequency domain equalization for obtaining the frequency domain data information requires the complexity $O\left[N^{2}\right]$.

In the conventional OLA-MMSE-FDE and FASTMMSE-FDE methods for the TS-OFDM signals, the Maclaurin's expansion algorithm is employed for the approximation of inverse matrix calculation [14]. The construction of CFR matrix and the received frequency domain signal matrix for the conventional OLA-MMSEFDE and FAST-MMSE-FDE methods require the complexity $O\left[N \log _{2} N+3 N\right]$ and $O\left[P^{2}+3 P\right]$, respectively. In [14], the order of total complexity both for the inverse matrix calculation with the order of Maclaurin's expansion of $Q_{2}$ and the equalization in the frequency domain are given by $O\left[2 Q_{2} N \log _{2} N+\left(2 Q_{2}+3\right) N\right]$ for the OLA-MMSE-FDE method and $O\left[N^{2}+\left(2 Q_{2}+1\right) P^{2}+\right.$ $\left.\left(2 Q_{2}+3\right) P\right]$ for the FAST-MMSE-FDE method, respectively. Here it should be noted that the order of complexities for $N$-points FFT (where $N$ is the power of 2) and $P$-points DFT (where $P$ is not the power of 2 ) are given by $O[N]$ and $O[P]$, respectively, in [14]. However, these complexities are given by $O\left[N \log _{2} N\right]$ and $O\left[P^{2}\right]$, respectively, in this paper. By using Table 1 , the comparisons of overall computation complexities between the conventional FDE and proposed TDE methods when assuming the actual parameters are evaluated in Section 4.

Table 1 Order of computation complexity for proposed TDE and conventional FDE methods

\begin{tabular}{|c|c|c|c|c|}
\hline \multirow[b]{2}{*}{ Methods } & \multicolumn{4}{|c|}{ Equalization methods with direct inverse matrix calculation } \\
\hline & $\begin{array}{l}\text { Removing ISI } \\
\text { from TS }\end{array}$ & $\begin{array}{l}\text { Construction of channel matrix } \\
\text { and received signal matrix }\end{array}$ & $\begin{array}{l}\text { Calculation of inverse } \\
\text { matrix }\end{array}$ & Equalization \\
\hline MMSE-FDE [9] & N/A & $O\left[N^{3}+2 N^{2} \log _{2} N+N^{2}+N \log _{2} N\right]$ & $O\left[N^{3}\right]$ & $O\left[N^{2}\right]$ \\
\hline OLA-MMSE-FDE [14] & $O\left[S^{2}-S\right]$ & $O\left[N^{3}+2 N^{2} \log _{2} N+N^{2}+N \log _{2} N\right]$ & $O\left[N^{3}\right]$ & $O\left[N^{2}\right]$ \\
\hline FAST-MMSE-FDE [14] & $O\left[S^{2}-S\right]$ & $O\left[P^{3}+2 S P^{2}+2 P^{2}\right]$ & $O\left[P^{3}\right]$ & $O\left[P^{2}+N^{2}\right]$ \\
\hline \multirow[t]{2}{*}{ Proposed TDE } & $O\left[S^{2}-S\right]$ & $O\left[N S^{2}+N S\right]$ & $O\left[N^{3}\right]$ & $\mathrm{O}\left[\mathrm{N}^{2}+\mathrm{Nlog}_{2} N\right]$ \\
\hline & \multicolumn{4}{|c|}{ Equalization methods with complexity reduction algorithms } \\
\hline \multirow{2}{*}{ MMSE-FDE [10] } & \multirow{2}{*}{ N/A } & $O\left[\left(2 Q_{1}+2\right) N \log _{2} N+N\left(Q_{1}+1\right)^{2}\right.$ & \multirow{2}{*}{$O\left[3 N\left(Q_{1}+1\right)^{2}\right]$} & \multirow{2}{*}{$O\left[N^{2}\right]$} \\
\hline & & $\left.+N\left(Q_{1}+1\right)+N \log _{2} N\right]$ & & \\
\hline OLA-MMSE-FDE [14] & $O\left[S^{2}-S\right]$ & $O\left[N \log _{2} N+3 N\right]$ & \multicolumn{2}{|c|}{$\mathrm{O}\left[2 \mathrm{Q}_{2} \mathrm{~N} \log _{2} N+\left(2 \mathrm{Q}_{2}+3\right) \mathrm{N}\right]$} \\
\hline FAST-MMSE-FDE [14] & $O\left[S^{2}-S\right]$ & $O\left[P^{2}+3 P\right]$ & \multicolumn{2}{|c|}{$O\left[N^{2}+\left(2 Q_{2}+1\right) P^{2}+\left(2 Q_{2}+3\right) P\right]$} \\
\hline Proposed TDE & $O\left[S^{2}-S\right]$ & $O\left[N S^{2}+N S\right]$ & $\mathrm{O}\left[3 N S^{2}\right]$ & $O\left[N^{2}+N \log _{2} N\right]$ \\
\hline
\end{tabular}

$N$ is the number of FFT/IFFT points, $S$ is the length of TS, $Q_{1}$ is the bandwidth of CFR matrix [10], $Q_{2}$ is the order of Maclaurin's expansion [14], and $P=N+S-1$ 


\section{Performance evaluations}

In this section, various computer simulation results are presented for the proposed TDE method as comparing with various conventional FDE methods both for the CPOFDM and TS-OFDM signals. The simulation parameters to be used in the following evaluations are listed in Table 2. In the conventional MMSE-FDE with the parallel block inverse matrix algorithm for the CP-OFDM signal, the CFR matrix is approximated by the banded matrix with the lower and upper bandwidth of $Q_{1}=2$ as used in [10] and $Q_{1}=15$ which is the same bandwidth for the proposed TDE method with the symmetric banded matrix. In the conventional FDE with the OLA-MMSEFDE and FAST-MMSE-FDE methods for the TS-OFDM signals, the order of Maclaurin's expansion in the calculation of inverse matrix is set to $Q_{2}=1$ as using in [14]. The Rayleigh multipath fading channel is assumed by Jake's Doppler spectrum model with an exponential power delay profile having the power decay constant $-1 \mathrm{~dB}$ and the length of delay path $L=14$. The length of TS both for the proposed and conventional TS-OFDM signals is taken by $S=16$ so as to be longer than the length of delay path $L=14$. In the following evaluations, the normalized Doppler frequency $R_{\mathrm{D}}=f_{\mathrm{d} \max } / \Delta f$ (\%) is employed as the measure of mobile environments in which $f_{\mathrm{dmax}}$ is the maximum Doppler frequency and $\Delta f$ is the OFDM subcarrier spacing.

Table 2 Simulation parameters

\begin{tabular}{ll}
\hline Parameter & Value \\
\hline Number of data subcarriers $(N)$ & 128 \\
Number of FFT/IFFT points $(N)$ & 128 \\
Length of TS for TS-OFDM $(S)$ & 16 \\
Length of $G$ l for CP-OFDM $\left(N_{g}\right)$ & 16 \\
Bandwidth of banded matrix $\left(Q_{1}\right)$ & 2 and 15 \\
Order of Maclaurin's expansion $\left(Q_{2}\right)$ & 1 \\
Number of symbols per one frame $(M)$ & 64 \\
Allocated OFDM frequency bandwidth $(W)$ & $2 \mathrm{MHz}$ \\
OFDM subcarrier spacing ( $\Delta f$ ) & $78.125 \mathrm{kHz}$ \\
Modulation method for data subcarriers & $\mathrm{QPSK}$ \\
Radio frequency & and $16 \mathrm{QAM}$ \\
$\quad$ Rayleigh multipath fading channels model & $5 \mathrm{GHz}$ \\
Power delay profile & \\
Decay constant & Exponential \\
Number of delay paths $(L)$ & $-1 \mathrm{~dB}$ \\
Number of scattered rays per one delay path & 14 \\
Normalized Doppler frequency $\left(R_{\mathrm{D}}\right)$ & 20 \\
\hline
\end{tabular}

Figure 3 shows the comparison of power spectrum density (PSD) at the output of transmitter for the conventional CP-OFDM, the TS-OFDM with the PN sequence, and the proposed TS-OFDM with the new design of TS signal when the modulation method for data subcarriers is QPSK and the oversampling ratio of OFDM transmission signal is taken by four times. From the figure, it can be seen that the PSD for the conventional TS-OFDM with the PN sequence has higher leakage of PSD at the outside of allocated OFDM frequency bandwidth. This is the reason that the same data pattern of PN sequence is used for all data symbols over one frame and the time domain PN sequence itself has wider frequency bandwidth as compared with that for the conventional CP-OFDM signal. While the PSD for the proposed TS-OFDM of using different patterns of TS signals with the triangular window function shows almost the same PSD as that for the conventional CP-OFDM signal. From the figure, it can be concluded that the proposed TS-OFDM with the new design of TS signal can be used in the CIR estimation with keeping lower leakage of PSD at the outside of allocated OFDM frequency bandwidth.

Figure 4 shows the CIR estimation accuracy at every sampling time both for the conventional TS-OFDM with the PN sequence and the proposed TS-OFDM with the new design of TS signal when changing the normalized Doppler frequency $\left(R_{\mathrm{D}}\right)$ and $C / N$. The CIR estimation accuracy is evaluated by the normalized mean square error (NMSE). Here it should be noted that the estimated CIR at every sampling time is obtained by using the cubic interpolation method for the estimated CIR at every symbol. From the figure, it can be observed that the accuracy of estimated CIR at every sampling time for the proposed

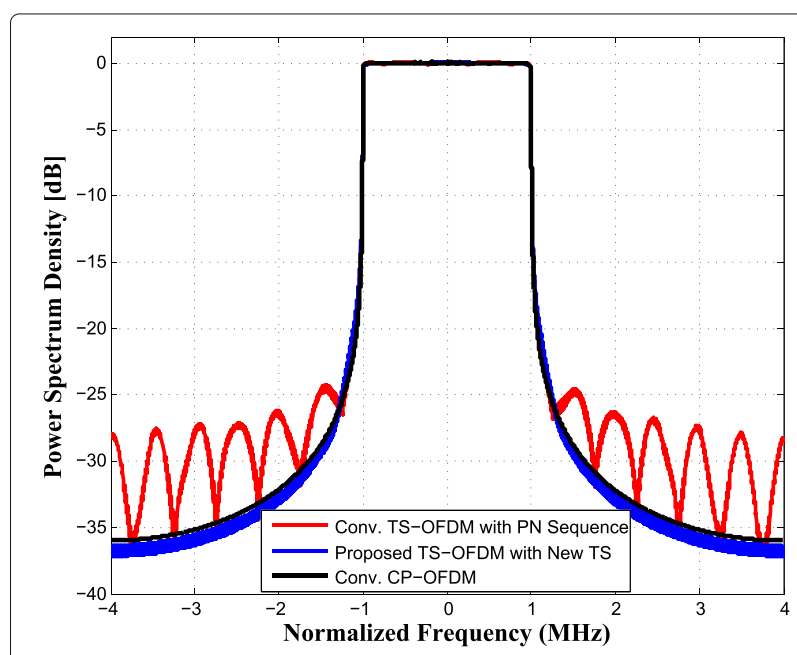

Fig. 3 Power spectrum density for proposed TS-OFDM signal with new design of TS, conventional TS-OFDM signal with PN sequence, and conventional CP-OFDM signal 


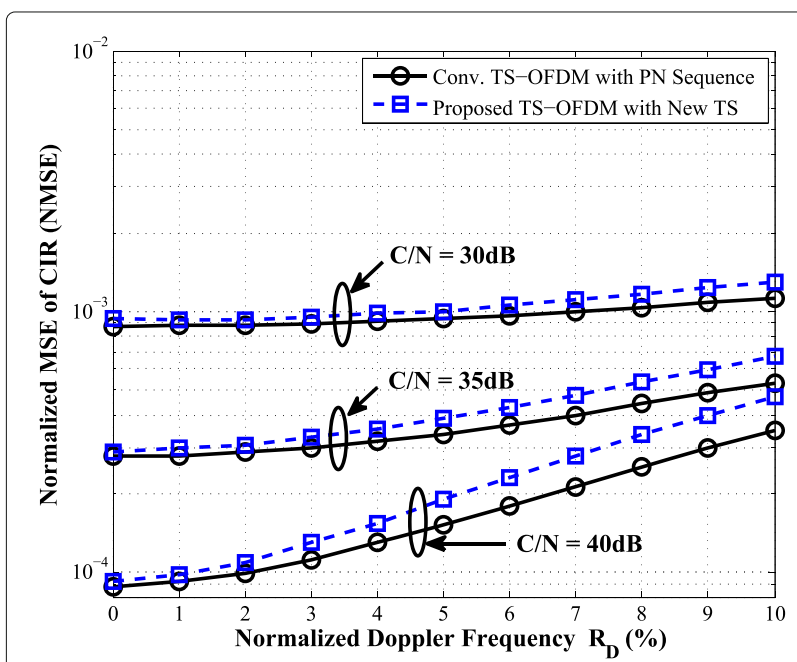

Fig. 4 CIR estimation accuracy (NMSE) for proposed and conventional TS-OFDM when changing normalized Doppler frequency $R_{D}$ and $C / N$

TS signal is almost the same performance as that for the PN sequence. From Figs. 3 and 4, it can be concluded that the estimated CIR of using the proposed new design of TS signal can be used in the removing of ISI by using (12) and the construction of both the received time domain signal matrix $\left[b\left(m, n_{3}\right)\right]$ and the time domain CIR matrix $\left[A_{m}\left(n_{3}, n\right)\right]$ by using (19) and (20) precisely with keeping lower leakage of PSD at the outside of OFDM allocated bandwidth even in higher time-varying fading channels.

Figure 5 shows the BER performances of the proposed TDE method for the TS-OFDM signal with the parallel block inverse matrix algorithm when changing the normalized Doppler frequency $\left(R_{\mathrm{D}}\right)$. The modulation method

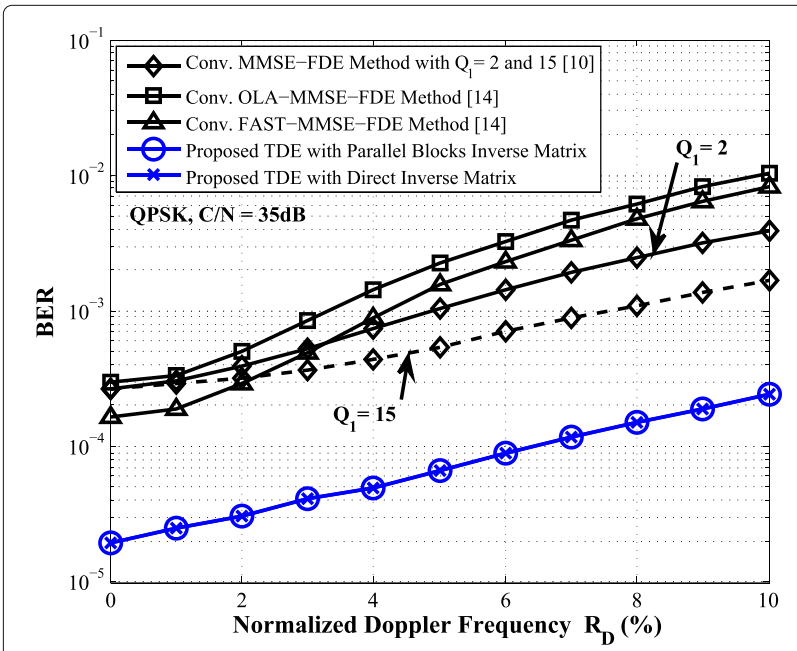

Fig. 5 BER performances versus the normalized Doppler frequency $R_{D}$ for proposed TDE and conventional FDE methods with low-complexity reduction algorithms when the modulation method is QPSK and C/N is $35 \mathrm{~dB}$ is QPSK and the $C / N$ is $35 \mathrm{~dB}$. In the figure, the conventional MMSE-FDE for CP-OFDM of using the approximated banded matrix with the lower and upper bandwidth of $Q_{1}=2$ and $Q_{1}=15$ [10], and the OLA-MMSEFDE and FAST-MMSE-FDE methods for TS-OFDM with the Maclaurin's expansion order of $Q_{2}=1$ [14] are also shown as the purpose of comparisons with the proposed TDE method. In the conventional MMSE-FDE for the CP-OFDM signal, the scattered pilot subcarriers in the frequency domain are usually employed in the estimation of CFR matrix. However, the estimation accuracy of CFR matrix by using the scattered pilot subcarriers would be degraded due to the occurrence of ICI for the received pilot subcarriers in higher time-varying fading channels [12]. From this fact, this paper assumes that the CIR estimated by the proposed TS-OFDM signal is employed in the construction of CFR matrix even for the conventional MMSE-FDE method for the CP-OFDM signal so as to compare the BER performance with the proposed TDE method fairly under the assumption of same channel estimation accuracy. From the figure, it can be observed that the proposed TDE method for the TS-OFDM signal with the parallel block inverse matrix algorithm shows much better BER performance than the conventional OLA-MMSE-FDE and FAST-MMSE-FDE methods with the Maclaurin's expansion method [14], and MMSE-FDE method [10] even when the banded matrix is approximated by $Q_{1}=15$. These are the reasons that the channel frequency matrix for the conventional MMSE-FDE method is generated by using the approximated banded matrix and for the OLA-MMSE and FAST-MMSE methods are generated assuming the linear approximation of CIR in the time-varying fading channels. These approximation and assumption would lead to the inevitable error in the calculation of inverse matrix especially in higher time-varying fading channels. Here it should be noted that the BER performance of proposed TDE method with the parallel block inverse matrix algorithm shows the same BER performance as that for using the direct inverse matrix calculation as explained in the sub-section 2.3 of Section 2.

Figures 6 and 7 show the BER performances versus $C / N$ for the proposed TDE method with the parallel block inverse matrix algorithm when the modulation method is QPSK and the normalized Doppler frequencies are $R_{\mathrm{D}}=3$ and $10 \%$ which correspond to lower and higher mobile environments, respectively. Here the normalized Doppler frequencies $R_{\mathrm{D}}=3$ and $10 \%$ correspond to the vehicle speeds at 100 and $340 \mathrm{~km} / \mathrm{h}$, respectively. In the figures, the BER performances of conventional lowcomplexity MMSE-FDE method for CP-OFDM of using the approximated banded matrix with the bandwidth of $Q_{1}=2$ and $Q_{1}=15$ [10], and the OLA-MMSE-FDE and FAST-MMSE-FDE methods for TS-OFDM with the 


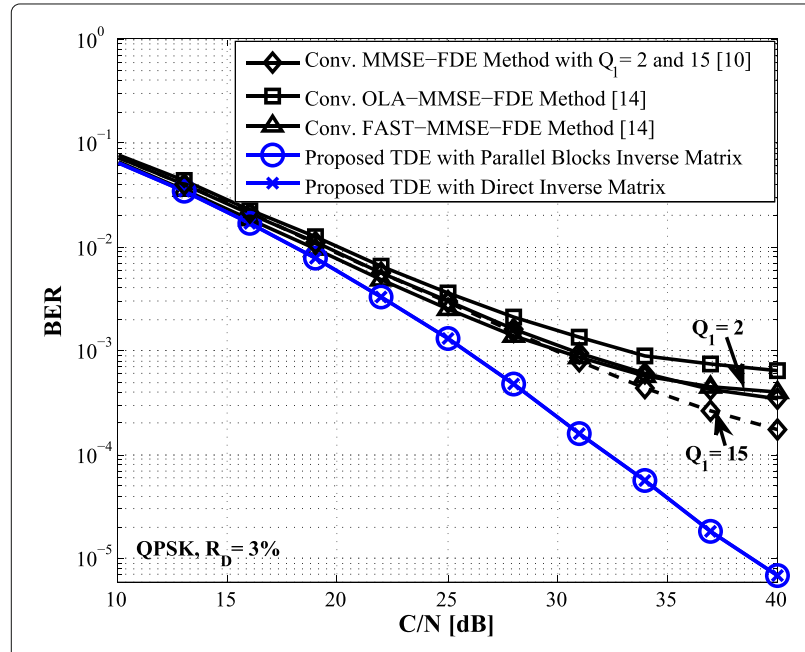

Fig. 6 BER performances versus $C / N$ for proposed TDE and conventional FDE methods with low-complexity reduction algorithms when the modulation method is QPSK and $R_{\mathrm{D}}$ is $3 \%$

Maclaurin's expansion order of $Q_{2}=1[14]$ are also shown as the purpose of comparisons with the proposed TDE method. From Figs. 6 and 7, it can be observed that the proposed method shows much better BER performance than the conventional methods under both lower and higher mobile environments. However, the conventional methods show better BER performance only under lower mobile environments because of employing the approximation in the construction of CFR matrix so as to reduce the computation complexity. The proposed TDE method also shows better BER performance than the conventional MMSE-FDE method even when the banded matrix is approximated by $Q_{1}=15$ which corresponds to the same computation complexity as the proposed TDE method.

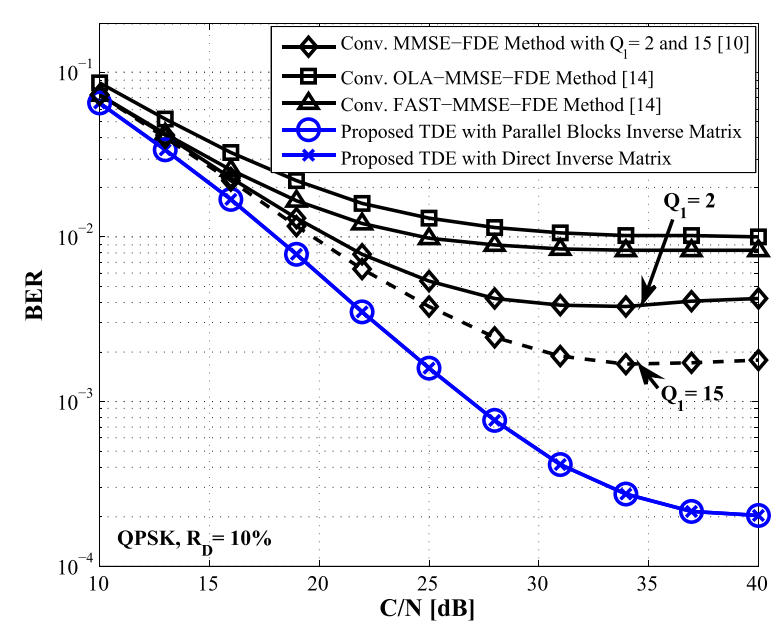

Fig. 7 BER performances versus $C / N$ for proposed TDE and conventional FDE methods with low-complexity reduction algorithms when the modulation method is QPSK and $R_{\mathrm{D}}$ is $10 \%$
Figure 8 shows the BER performances versus $C / N$ for the proposed TDE method when the modulation method is 16QAM and the normalized Doppler frequency $R_{\mathrm{D}}$ is $6 \%$ which correspond to the vehicle speed at $200 \mathrm{~km} / \mathrm{h}$. From the figure, it can be concluded that the proposed TDE method with the low-complexity parallel block inverse matrix algorithm can achieve much better BER performance than the conventional FDE methods of using the complexity reduction methods even when employing higher efficient modulation method of 16QAM.

Figure 9 shows the overall computation complexities for the conventional FDE methods and proposed TDE method when changing the number of FFT/IFFT points $(N)$. The overall computation complexities include the processing loads for the removing of ISI from the TS, construction of channel matrix, calculation of inverse matrix, and equalizations in the frequency and time domains as shown in Table 1. In the complexity evaluations, the lower and upper bandwidth employed in the approximation of banded CFR matrix for the conventional MMSE-FDE are evaluated by $Q_{1}=2$ as using in [10] and $Q_{1}=15$ which corresponds to the same bandwidth as that for the proposed TDE with the parallel block inverse matrix algorithm. From the figure, it can be observed that the overall complexities for all methods of using the direct inverse matrix calculation are much higher than that for using the complexity reduction methods especially as increasing $N$. When $N$ is 128 , the order of complexity for the conventional MMSE-FDE with the parallel block inverse matrix algorithm with $Q_{1}=15$ and the FAST-MMSE-FDE of using the Maclaurin's expansion method with $Q_{2}=1$ [14] are almost the same complexity as that for the proposed TDE method. However, the order of complexity for the conventional MMSE-FDE with $Q_{1}=2$ and the

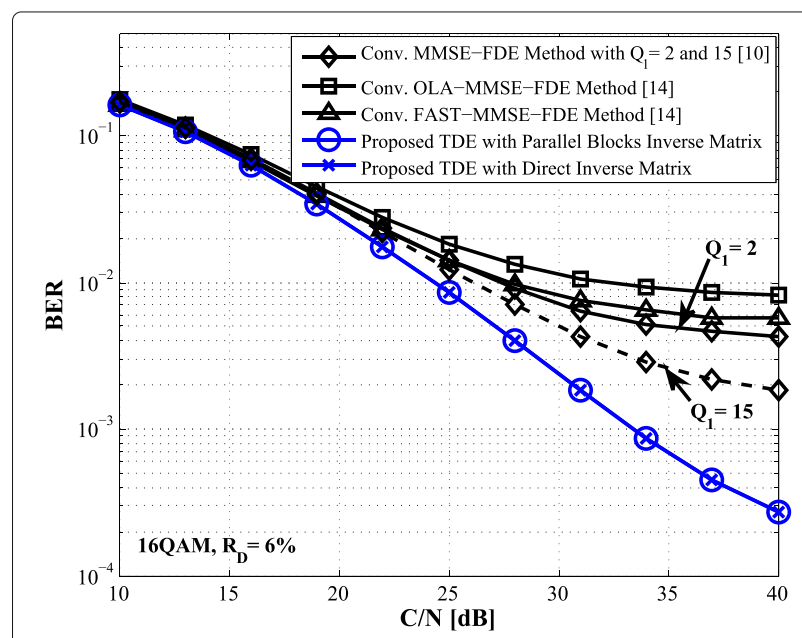

Fig. 8 BER performances versus $C / N$ for proposed TDE and conventiona FDE methods with low-complexity reduction algorithms when the modulation method is $16 \mathrm{QAM}$ and $R_{\mathrm{D}}$ is $6 \%$ 


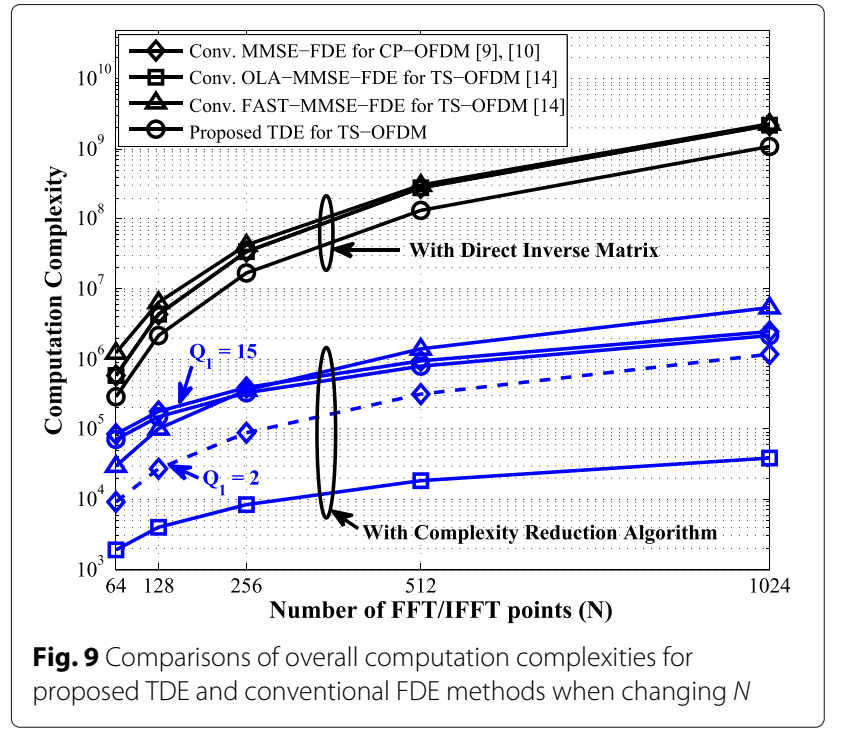

OLA-MMSE-FDE with $Q_{2}=1$ are lower than the proposed TDE with the parallel block inverse matrix algorithm. However, these computation complexity reductions for the conventional methods can achieve at the cost of considerable degradation of BER performances as shown in Figs. 5, 6, 7 and 8.

From these results, it can be concluded that the proposed TDE method with the parallel block inverse matrix algorithm can achieve much better BER performance than the conventional FDE methods of using the complexity reduction methods with the allowable increase of computation complexity.

\section{Conclusions}

This paper proposed the time domain equalization (TDE) method for the TS-OFDM signal in higher time-varying fading channels. The salient features of the proposed method are to employ the new design of TS signal in the estimation of CIR at every sampling time and the time domain equalization method of using the parallel block inverse matrix algorithm. To demonstrate the effectiveness of the proposed TDE method, this paper conducted various computer simulations. From the simulation results, this paper confirmed that the proposed TS signal can achieve lower leakage of power spectrum density at the outside of allocated OFDM frequency bandwidth with keeping higher CIR estimation accuracy. This paper also concluded that the proposed TDE method with the parallel block inverse matrix algorithm can achieve much better BER performance than the conventional FDE methods of using the complexity reduction methods with allowable increase of computation complexity in higher time-varying fading channels.

\section{Acknowledgements}

The authors would like to thank to the Japanese Government

(Monbukagakusho: MEXT) Scholarships who has supported this research.

\section{Competing interests}

The authors declare that they have no competing interests.

Received: 24 March 2016 Accepted: 7 December 2016

Published online: 03 January 2017

\section{References}

1. YG Li, GL Stuber, Orthogonal Frequency Division Multiplexing for Wireless Communications. (Springer, Georgia, 2006)

2. JG Proakis, M Salehi, Digital Communications, 5th ed. (McGrawHill, New York, 2008)

3. F Hlawatsch, G Matz, Wireless Communications Over Rapidly Time-Varying Channels. (Academic Press, Vienna, 2011)

4. M Liu, M Crussiere, J Helard, A novel data-aided channel estimation with reduced complexity for TDS-OFDM systems. IEEE Trans. Broadcast. 58(2), 247-260 (2012)

5. T Yamamoto, F Adachi, Training sequence inserted OFDM transmission with MMSE-FDE. IEICE Trans. Commun. E97-B(2), 476-483 (2014)

6. HC Wu, Analysis and characterization of intercarrier and interblock interferences for wireless mobile OFDM systems. IEEE Trans. Broadcast. 52(2), 203-210 (2006)

7. Y Zhao, SG Haggman, Intercarrier interference self-cancellation scheme for OFDM mobile communication systems. IEEE Trans. Commun. 49(7), 1185-1191 (2001)

8. MX Chang, A novel algorithm of inter-subchannel interference selfcancellation for OFDM systems. IEEE Trans. Wireless Commun. 6(8), 2881-2893 (2007)

9. YS Choi, PJ Volts, FA Cassara, On channel estimation and detection for multicarrier signals in fast and selective Rayleigh fading channels. IEEE Trans. Commun. 49(8), 1375-1387 (2001)

10. L Rugini, P Banelli, G Leus, Simple equalization of time-varying channels for OFDM. IEEE Comm. Lett. 9, 619-621 (2005)

11. D Shimbo, N Maeda, H Mishima, J Ido, in Proceedings of IEEE 78th Vehicular Technology Conference (VTC-Fall). Inter-carrier interference compensation for zero padding OFDM (VTC, Las Vegas, 2013), pp. 1-5

12. BK Engiz, C Kurnaz, H Sezgin, in Proceedings of 8th Wireless and Mobile Computing, Networking and Communications (WiMob). Performance analysis of pilot arrangement for OFDM systems over time varying frequency selective channels (WiMob, Barcelona, 2012), pp. 113-117

13. J Fu, CY Pan, ZX Yang, L Yang, Low-complexity equalization for TDS-OFDM systems over doubly selective channels. IEEE Trans. Broadcast. 51(3), 401-407 (2005)

14. J Hao, J Wang, Y Wu, A new equalizer in doubly-selective channels for TDS-OFDM. IEEE Trans. Broadcast. 61(1), 91-97 (2015)

15. L Lin, J Lu, L Ying, REW Car, Fast algorithm for extracting the diagonal of the inverse matrix with application to the electronic structure analysis of metallic systems. Commun. Math. Sci. 7(3), 755-777 (2009)

16. J Song, Z Yang, L Yang, K Gong, C Pan, J Wang, Y Wu, Technical review on Chinese digital terrestrial television broadcasting standard and measurements on some working modes. IEEE Trans. Broadcast. 53(1), 1-7 (2007)

17. AA Quadeer, in Proceedings of Wireless Communications, Networking and Inforomation Security (WCNIS). Enhanced Equalization in OFDM Systems Using Cyclic Prefix (WCNIS, Beijing, 2010), pp. 40-44

18. KMM Prabhu, Window Functions and Their Applications in Signal Processing (CRC Press, Boca Raton, 2013)

19. P Reangsuntea, P Boonsrimuang, K Mori, H Kobayashi, in Proceedings of 8th International conference on signal processing and communication systems (ICSPCS). Iterative Based Time Domain Equalization Method for OFDM Signal under High Mobile Environments (ICSPCS, Gold Coast, 2014), pp. 1-6 